\title{
Construction and validation of an N6-methyladenosine-associated prognostic signature in hepatocellular carcinoma
}

\author{
PENG ZHU ${ }^{1 *}$, QIANQIAN REN ${ }^{2,3^{*}}$, NAN HE$^{4}$, CHENG ZHOU $^{1}$, QIANNA JIN ${ }^{2,3}$ and ZHAO GONG ${ }^{1}$ \\ ${ }^{1}$ Department of Hepatobiliary Surgery, Wuhan No. 1 Hospital; ${ }^{2}$ Department of Radiology, Union Hospital, \\ Tongji Medical College, Huazhong University of Science and Technology; ${ }^{3}$ Hubei Province Key Laboratory \\ of Molecular Imaging; ${ }^{4}$ Cancer Center, Department of Gastrointestinal Surgery, Union Hospital, \\ Tongji Medical College, Huazhong University of Science and Technology, Wuhan, Hubei 430022, P.R. China
}

Received March 14, 2020; Accepted October 20, 2020

DOI: $10.3892 / \mathrm{ol} .2021 .12482$

\begin{abstract}
Hepatocellular carcinoma (HCC) is among the most common types of cancer that threat the public health worldwide. N6-methyladenosine (m6A) RNA methylation is associated with cancer initiation and progression, and is dynamically regulated by m6A RNA methylation-associated genes. However, little is known about the expression status and the prognostic value of m6A-associated genes in HCC. The present study aimed to identify the expression profiling pattern and clinical significance of m6A-associated genes in HCC. Consensus clustering analysis was performed to identify the clusters of HCC with different clinical outcomes. A prognostic signature built by the least absolute shrinkage and selection operator Cox regression model was utilized to discover subtypes associated with different clinical outcomes of patients with HCC in the discovery cohort from The Cancer Genome Atlas. The differences between subgroups were characterized in terms of epigenetic dysregulation and somatic mutation frequencies. The International Cancer Genome
\end{abstract}

Correspondence to: Dr Zhao Gong, Department of Hepatobiliary Surgery, Wuhan No. 1 Hospital, 215 Zhongshan Avenue, Wuhan, Hubei 430022, P.R. China

E-mail: 1072503223@qq.com

Dr Qianna Jin, Department of Radiology, Union Hospital, Tongji Medical College, Huazhong University of Science and Technology, 1277 Jiefang Avenue, Wuhan, Hubei 430022, P.R. China

E-mail: jinqianna2018@gmail.com

${ }^{*}$ Contributed equally

Abbreviations: HCC, hepatocellular carcinoma; m6A, N6-methyladenosine; TCGA, The Cancer Genome Atlas; GEO, Gene Expression Omnibus; HPA, Human Protein Atlas; LASSO, least absolute shrinkage and selection operator; DFS, disease-free survival; OS, overall survival; BCLC, Barcelona Clinic Liver Cancer; PPI, protein-protein interaction; $\mathrm{HR}$, hazard ratio

Key words: HCC, prognostic signature, survival analysis, m6A RNA methylation
Consortium cohort and two independent cohorts from the meta-Gene Expression Omnibus database were used for external validation. Most of the m6A-associated genes were upregulated and involved in the prognosis and malignancy of HCC. A four-gene prognostic signature revealed two HCC subtypes (namely, high- and low-risk group) that was associated with different clinical outcomes. Patients in the high-risk group were accompanied with increased epigenetic silencing and significant mutations in TP53 and FLG, while ALB was frequently mutated in the low-risk group. In conclusion, an m6A-based signature was constructed to predict the prognosis of patients with HCC, which may provide a tool for reliable prognosis assessment for clinicians, and aid clinical treatment decision-making.

\section{Introduction}

According to the International Agency for Research on Cancer, hepatocellular carcinoma (HCC) is the second leading cause of tumor-associated death worldwide (1). HCC commonly develops in patients with chronic hepatitis, such as viral hepatitis (2). Various treatments for HCC, including resection, transplantation and interventional therapy, have undergone immense progress over the last decades, but the prognosis of $\mathrm{HCC}$ remains poor in patients at the late stage (3). Additionally, the high rate of postsurgical recurrence and metastasis (50-70\% at 5 years) represents a major challenge, as this disease is highly refractory to conventional chemotherapy and radiation (4). Currently, the Barcelona Clinic Liver Cancer (BCLC) staging classification is the most extensively used classification system for HCC, which can be applied for the assessment of patient prognosis and the selection of appropriate therapies (5). However, it has been reported that patients with HCC with the same BCLC stage may include various tumor subtypes, such as nodular or infiltrating tumors, thus resulting in differences in treatment responses and survival (6). Therefore, it is critical to identify novel and reliable prognostic molecular signatures in HCC from basic and clinical research.

N6-methyladenosine (m6A), methylated at the N6 position of adenosine, is the most abundant epigenetic and evolutionarily conserved modification of mRNAs and non-coding RNAs in 
mammals (7-10). In total RNA, $0.1-0.4 \%$ of adenosines are modified by m6A methylation (11). m6A methylation affects almost every aspect of RNA metabolism, including abundance, alternative splicing, stability, nuclear export, decay and translation (12-14), thus negatively regulating protein expression in a post-translational manner. The identification of m6 A adenosine methyltransferases ('writers'), demethylases ('erasers') and binding proteins ('readers') revealed that m6A modification is reversible (15). Increasing evidence has indicated that the m6A modification may be involved in various physiological processes and diseases, including circadian rhythms, stem cell differentiation and maternal-to-zygotic transition (16), as well as the carcinogenesis of several types of tumor,including cervical cancer (17), prostate cancer (18), breast cancer (19), pancreatic cancer (20) and HCC (21). The characterization for m6A sparked a renewed interest in this particular RNA modification. However, its expression pattern, as well as its prognostic value, has not been fully elucidated in HCC.

In the present study, the m6A patterns were estimated based on the 20 widely reported m6A RNA regulators and were systematically characterized for the potential subtypes in a multiomics view, including somatic mutations and DNA methylation.

\section{Materials and methods}

Public data source. TCGA- Liver hepatocellular carcinoma (LIHC) cohort data, including RNA sequencing, mutation and clinical data, were downloaded from The National Cancer Institute Genomic Data Commons (https://portal.gdc.cancer. gov/). Maftools v2.4.12 (22) was utilized to infer significant cancer mutated genes using default parameters. Illumina Human Methylation 450 Beadchip (450K array; Illumina, Inc.) was used to measure the DNA methylation data. For a gene with $>1$ probe mapping to its promoter, the median $\beta$ value was considered. MethylMix v2.18.0 (23) was used to identify the expression of genes associated with methylation events. The International Cancer Genome Consortium (ICGC; https://icgc. org) Japan cohort with 203 patients with HCC and the meta-Gene Expression Omnibus (GEO) cohorts [GSE14520 (24) and GSE76427 (25); www.ncbi.nlm.nih.gov/geo] with 336 patients with HCC were utilized as the validation cohorts. In addition, validation of the translation of m6A-associated genes was performed using the Human Protein Atlas (HPA) database (version 19.2; http://www.proteinatlas.org).

Protein-protein interaction (PPI) network construction and correlation analysis. The PPI network among m6A RNA methylation regulators was constructed using Cytoscape v3.6.1 (www.cytoscape.org). Spearman correlation analysis was employed to reveal the correlation among different m6A RNA methylation regulators.

Consensus clustering analysis. To identify m6A patterns and classify patients for further analysis, patients with HCC in TCGA cohort were grouped using the ConsensusClusterPlus v1.52.0 (26) package, which was repeated 1,000 times to ensure the stability of classification. The optimal K value was determined according to consensus matrices, Consensus Cumulative Distribution Function and Delta Area.
Kaplan-Meier analysis (27) with log-rank test was performed to compare the survival of patients between clusters.

Screening of prognostic signatures and key prognostic genes. Univariate Cox proportional hazards regression was used to assess the independent m6A RNA methylation regulators, whose expression levels were significantly associated with the survival of patients. Hazard ratios (HRs) were used to identify protective $(\mathrm{HR}<1)$ or risk-associated genes $(\mathrm{HR}>1)$. LASSO-penalized Cox regression analysis performed using the glmnet v3.0-2 package (https://cran.r-project.org/package=glmnet) was used to achieve variable shrinkage and selection of key independent m6A RNA methylation regulators (28). An optimal model was determined based on a linear combination of the expression profiles of independent prognostic m6A RNA methylation regulators, weighted by the estimated regression coefficient derived from the LASSO Cox regression model coefficients multiplied with its mRNA expression level. The following formula was used to calculate the risk score of each patient: Risk score $=$ $\Sigma \mathrm{X} \mathrm{J} \times$ coef $\mathrm{J}$, where coef $\mathrm{J}$ is the coefficient, and $\mathrm{X} \mathrm{J}$ is the relative expression level of each gene standardized by Z-score. Subsequently, patients with HCC from TCGA were divided into high- and low-risk groups, according to the optimal cut-off value of risk scores obtained from the survminer v0.4.6 package (https://cran.r-project.org/package=survminer). Cox regression analysis was used to evaluate the association between risk score and disease-free survival (DFS) or overall survival (OS), in which age, sex, Tumor-Node-Metastasis (TNM) stage (29), Neoplasm_cancer_status and grade were used as covariates. Neoplasm_cancer_status is a statement about the progression (or not) of the original disease, whereas 'normal' is a statement that there was no disease to begin with. The Kaplan-Meier survival analysis with log-rank test and receiver operating characteristic (ROC) curve analysis were used to validate the multigene prognostic signature.

Gene Set Enrichment Analysis (GSEA). GSEA was performed to detect the significantly different signaling pathways in the set of expressed genes between the highand low-risk groups in the enrichment of the MSigDB Collection (c2.all.v7. 0. Symbols; https://www.gsea-msigdb. org/gsea/msigdb/collections.jsp\#C2). Gene set permutations were performed 1,000 times for each analysis. The Benjamini-Hochberg method was used to correct the P-values for multiple testing. The normalized enrichment score (NES), nominal P-value and false discovery rate (FDR) q-value indicated the significance of the association between gene sets and signaling pathways. $|\mathrm{NES}|>1, \mathrm{FDR} \leq 0.25$ and $\mathrm{P}<0.05$ were considered to indicate a statistically significant difference.

Statistical analysis. Data analysis was performed using R v3.6 software (https://cran.r-project.org/src/base/R-3/). All statistical tests were two-sided. The Wilcoxon signed-rank test and Mann-Whitney $U$ test were used for paired and unpaired samples, respectively, when the population with non-normal distribution or uneven variance. Cox regression analysis was performed to identify whether the final prognostic model was independent of traditional clinical features (including age, sex, TNM stage and histological grade). The Kaplan-Meier survival analysis with log-rank test and ROC curve analysis were used 


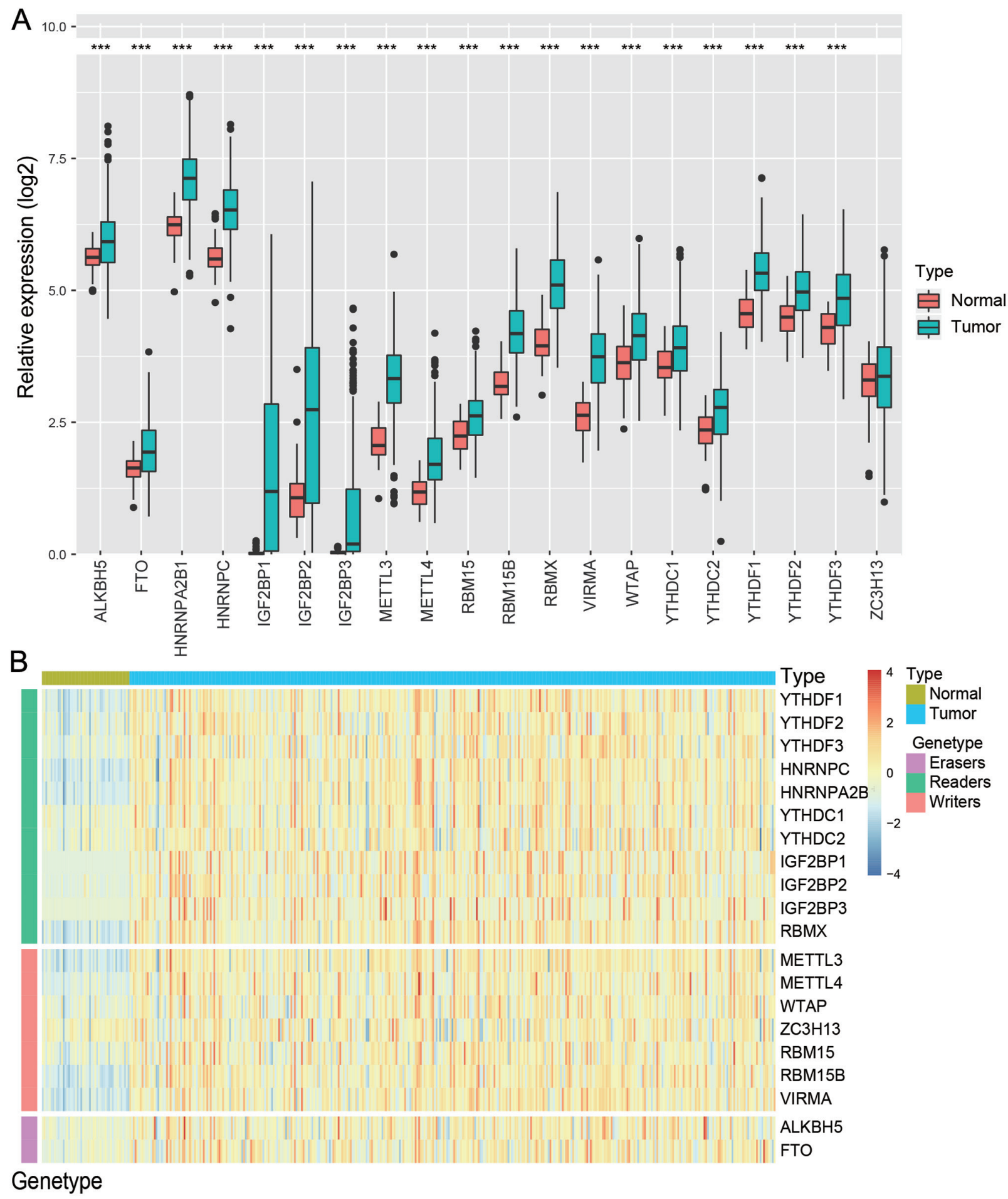

Figure 1. Landscape of m6A RNA methylation regulators in HCC. (A) Boxplot shows the median expression of 20 m6A RNA methylated regulatory factors in HCC samples compared with the control ones. ${ }^{* * *} \mathrm{P}<0.001$. (B) Expression heat map of $20 \mathrm{~m} 6 \mathrm{~A}$ RNA methylated regulatory factors between tumor and normal control samples in The Cancer Genome Atlas-LIHC cohort. HCC, hepatocellular carcinoma; m6A, N6-methyladenosine; LIHC, Liver HCC.

to assess the predictive ability of the prognostic model. $\mathrm{P}<0.05$ was considered to indicate a statistically significant difference.

\section{Results}

Landscape of $m 6 A$ RNA methylation regulators in $H C C$. The mRNA expression levels of the known m6A-associated regulators were analyzed in TCGA cohort, including m6A 'writers', such as METTL3, METTL4, WTAP, ZC3H13,
RBM15, RBM15B and VIRMA, m6A 'readers', such as YTHDF1, YTHDF2, YTHDF3, YTHDC1, YTHDC2, HNRNPC, HNRNPA2B1, IGF2BP1, IGF2BP2, IGF2BP3 and RBMX, and m6A 'erasers', such as FTO and ALKBH5. Compared with normal liver tissues, patients with HCC generally exhibited a higher proportion of m6A genes, except ZC3H13 (Fig. 1A and B). Furthermore, the expression levels of these aberrant m6A-associated genes were validated in the HPA database (Fig. S1). Notably, METTL3, WTAP, RBM15B, 
A
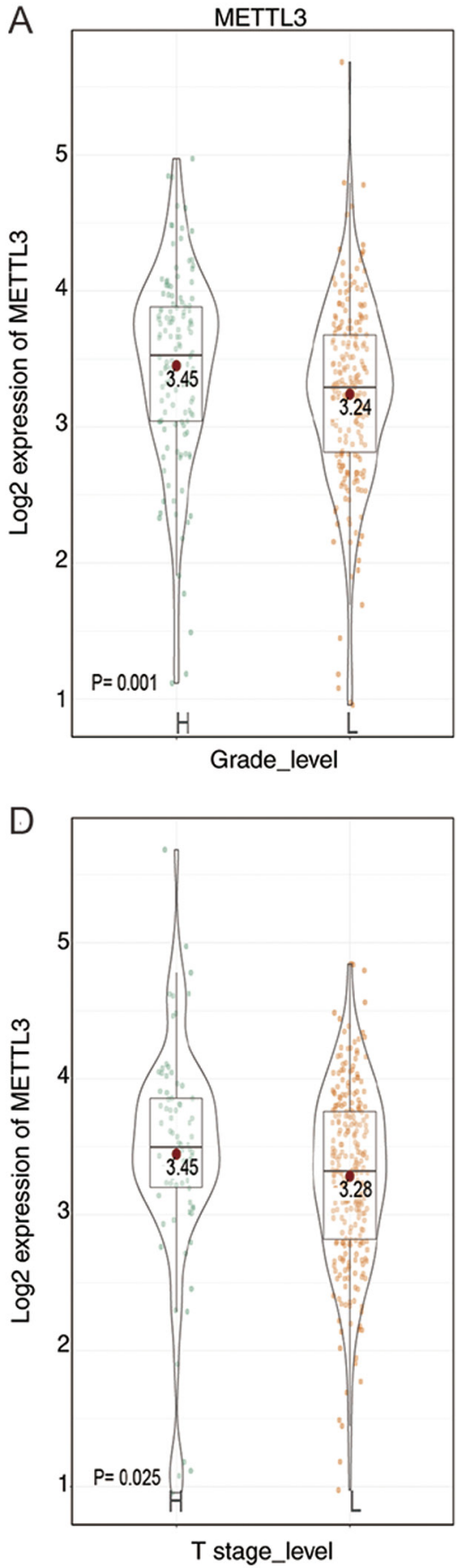

B

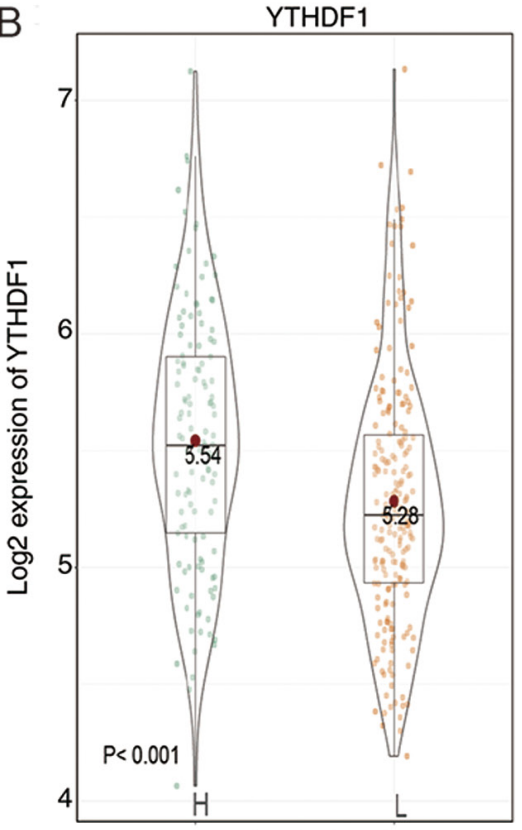

Grade_level

E

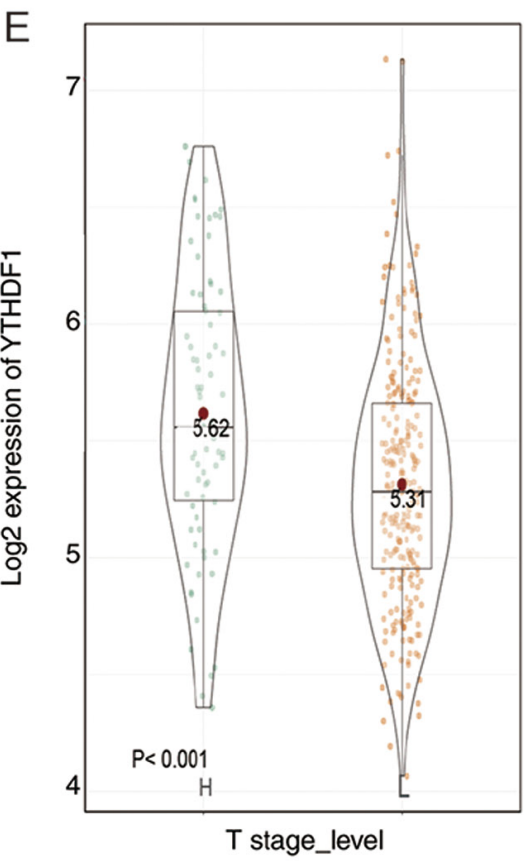

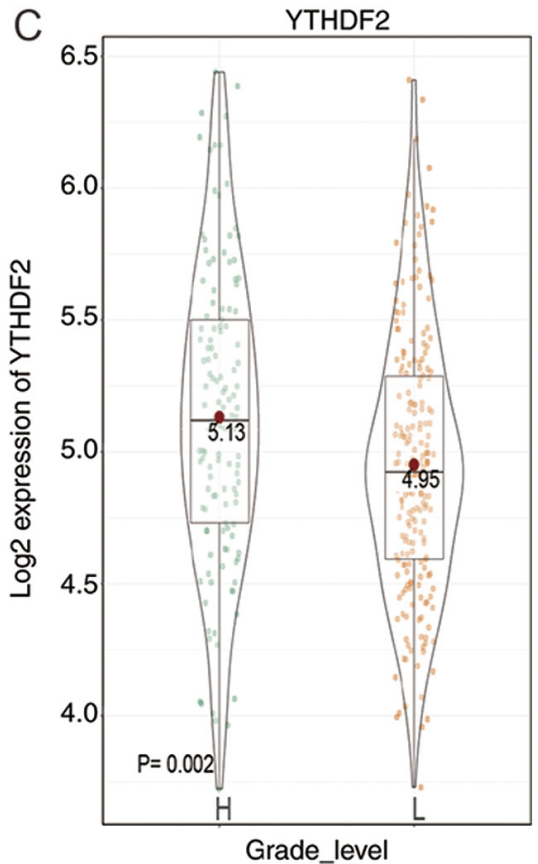

$\mathrm{F}$

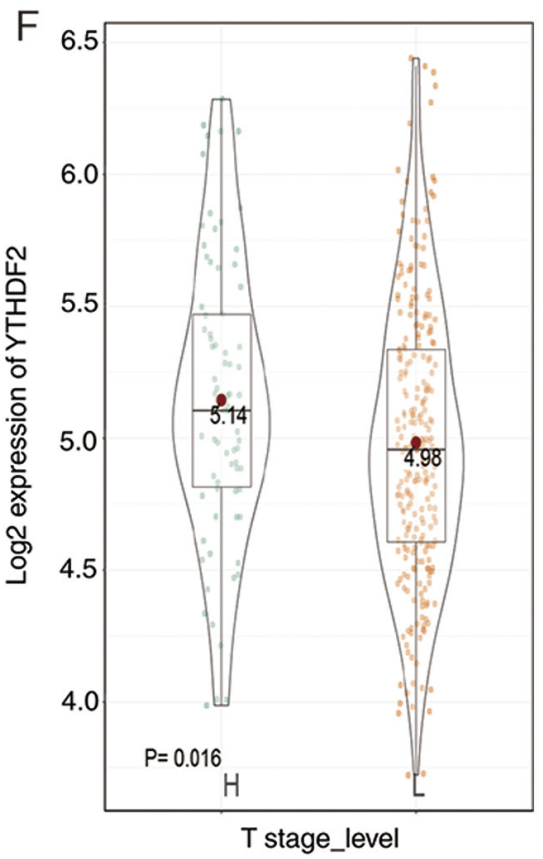

Figure 2. Association between m6A RNA methylation regulators and pathological grade/T stage. Expression levels of (A) METTL3, (B) YTHDF1 and (C) YTHDF2 in HCC with different pathological grades. Patients with G1/G2 pathological grade were divided into the L group, and patients with G3/G4 grade were divided into the H group. Expression levels of (D) METTL3, (E) YTHDF1 and (F) YTHDF2 in HCC with different T stages. Patients with T1/T2 stage were divided into the L group, and patients with T3/T4 stage were divided into the H group. m6A, N6-methyladenosine; HCC, hepatocellular carcinoma; T stage, tumor stage; H, high; L, low.

YTHDF1, YTHDF2, YTHDF3, HNRNPA2B1, IGF2BP1, IGF2BP2 and IGF2BP3 were absent from the HPA database. Overall, these data confirmed the highly significant dysregulation of several m6A-associated regulators in human HCC. The associations between each individual m6A RNA methylation regulator and the pathological features of $\mathrm{HCC}$ were further investigated. Patients with G1/G2 pathological grade were divided into the low (L) grade group, and patients with G3/G4 stage were divided into the high $(\mathrm{H})$ grade group. The expression levels of HNRNPA2B1, HNRNPC, IGF2BP1, IGF2BP2, IGF2BP3, RBM15B, RBM15, RBMX, METTL4, VIRMA,
YTHDC1, METTL3, YTHDF1 and YTHDF2 were higher in the $\mathrm{H}$ group compared with in the $\mathrm{L}$ group. Patients with tumor (T)1/T2 stage were divided into the L stage group, and patients with T3/T4 stage were divided into the H stage group. The expression levels of HNRNPA2B1, METTL4, RBM15B, RBMX, YTHDC1, YTHDF3, METTL3, YTHDF1 and YTHDF2 were higher in the $H$ group compared with in the L group. In summary, the expression levels of HNRNPA2B1, METTL3, METTL4, RBMX, YTHDF1 and YTHDF2 were significantly increased as the pathological grade and T-stage increased (Figs. 2 and S2). 


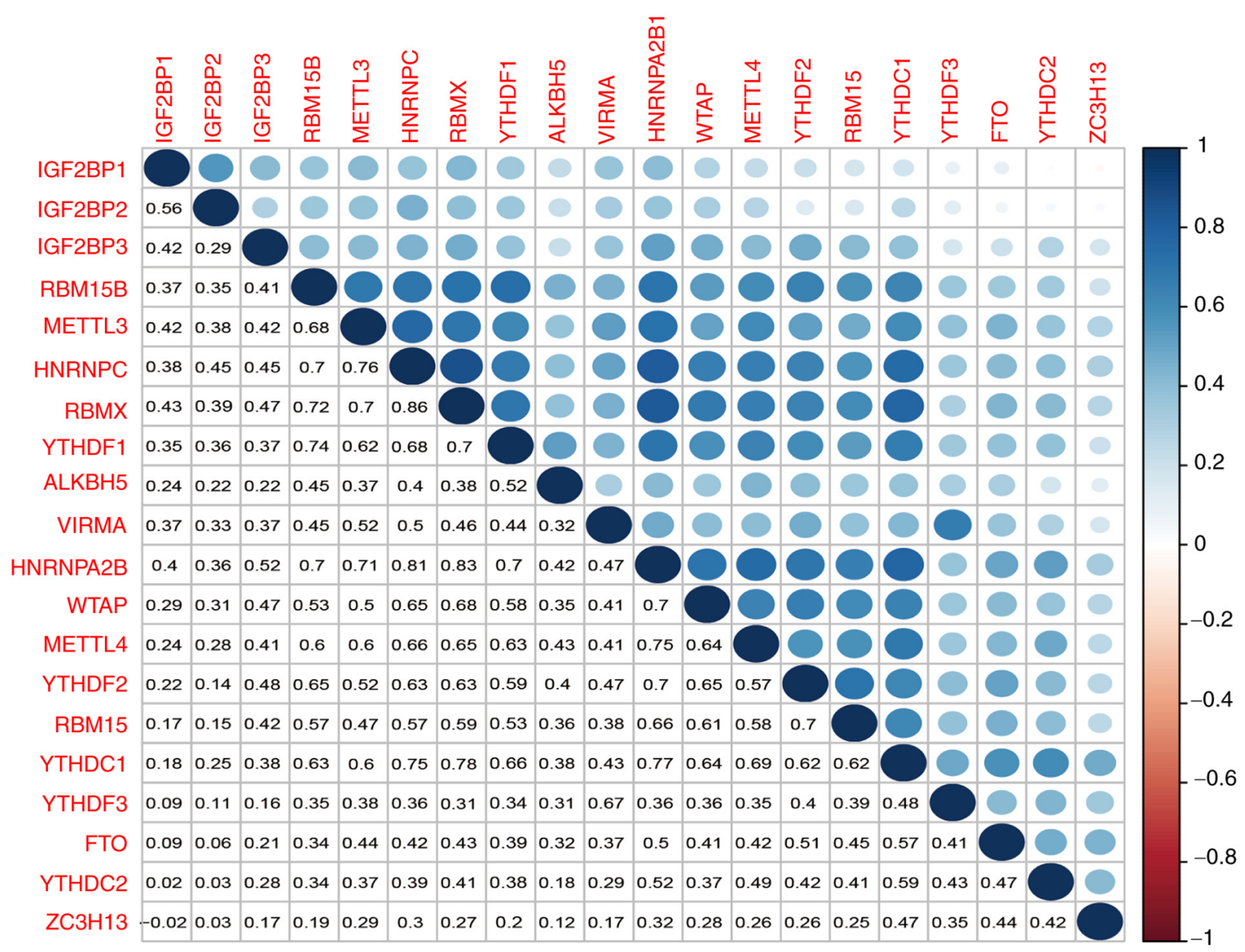

Figure 3. Correlation of the 20 N6-methyladenosine modification regulators estimated using Spearman's correlation analysis. The greater the absolute value of the correlation coefficient, the stronger the linear correlation. The color and size of the circles represent the characteristics of correlation. The larger the circle and the darker the color, the stronger the correlation. Blue represents a positive correlation, while red represents a negative correlation.

Fig. 3 shows that the majority of m6A RNA methylation regulators was positively correlated with each other, and the correlation between HNRNPC and RBMX was the most significant. Moreover, the PPI network depicted a comprehensive landscape of the interactions of m6A RNA methylation regulators and the writers, including WTAP, VIRMA and METTL4, ranked first according to the degree of connectivity (Fig. S3). The correlation coefficients between WTAP and IGF2BP3, YTHDF2, METTL3 and YTHDF1 were 0.47, $0.65,0.50$ and 0.58 , respectively. The correlation coefficients between VIRMA and IGF2BP3, YTHDF2, METTL3 and YTHDF1 were $0.37,0.47,0.52$ and 0.44 , respectively. The correlation coefficients between METTL14 and IGF2BP3, YTHDF2, METTL3 and YTHDF1 were 0.41, 0.57, 0.60 and 0.63 , respectively (Fig. 3).

Association between $\mathrm{m} 6 \mathrm{~A}$ regulators and HCC prognosis. Consensus Clustering is a method that provides quantitative evidence for determining the number and membership of possible clusters within a dataset, and to assess the stability of the discovered clusters (30). The expression similarity of $\mathrm{m} 6 \mathrm{~A}$ regulators and clustering stability was assessed using the ConsensusClusterPlus package. The cohort of patients with $\mathrm{HCC}$ was divided into three clusters, namely cluster 1, cluster 2 and cluster 3 (Fig. 4A-C). Fig. 4A shows the heatmaps of the consensus matrices for $\mathrm{k}=3$. Fig. 4B shows the cumulative distribution functions of the consensus matrix for each $\mathrm{k}$, estimated by a histogram of 100 bins. Fig. 4C shows the relative change in area under the CDF curve comparing $\mathrm{k}$ and $\mathrm{k}-1$. For $\mathrm{k}=2$, there is no $\mathrm{k}-1$, so the total area under the curve rather than the relative increase is plotted. The patients were divided into three clusters and there was no significant difference in the number of samples in each cluster. Notably, survival analysis revealed that cluster 1 was significantly associated with an improved DFS and cluster 2 with a poor DFS, while cluster 3 was characterized by an intermediate prognosis (Fig. 4D). Additionally, a favorable prognostic trend for OS was observed, although not statistically significant (data not shown), partly due to the limitation of the cohort size.

Construction and validation of the m6A-based HCC prognosis signature. Subsequently, the possible prognostic power of m6A RNA methylation regulators in HCC was analyzed by performing univariate Cox regression analysis. The results demonstrated that HNRNPA2B1, HNRNPC, IGF2BP3, METTL3, METTL4, RBM15, RBM15B, RBMX, VIRMA, WTAP, YTHDC1, YTHDC2, YTHDF1 and YTHDF2 were significantly associated with DFS, and HNRNPA2B1, IGF2BP3, METTL3, WTAP, YTHDF1 and YTHDF2 were 
A

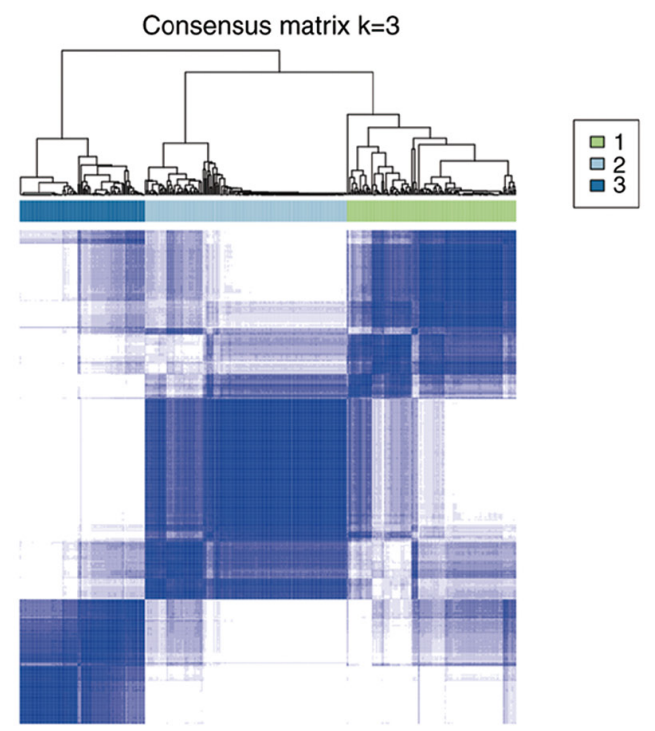

C

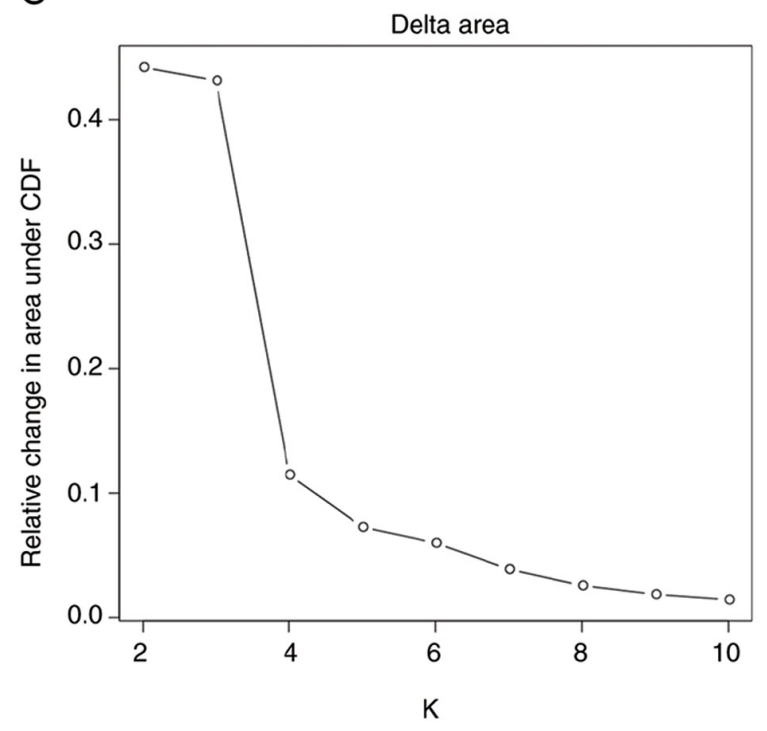

$\mathrm{B}$

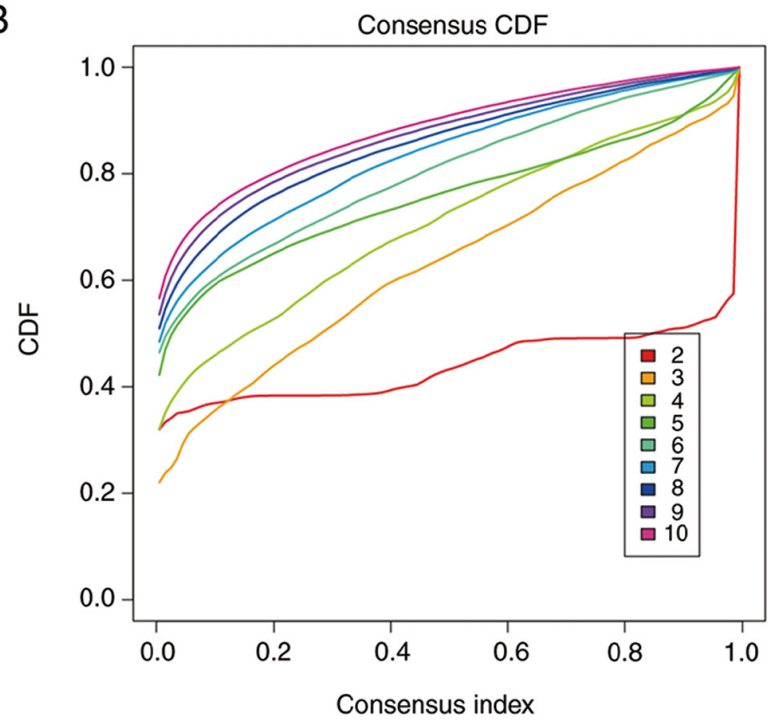

D $\quad$ Disease-free survival

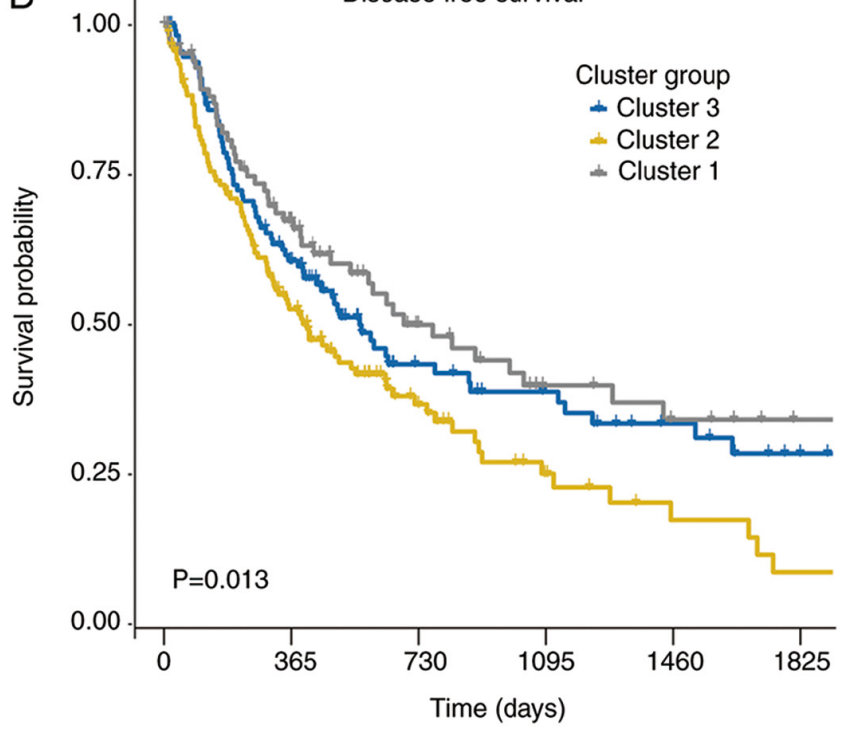

Figure 4. Differential DFS of The Cancer Genome Atlas-LIHC patients in the three different clusters. (A) At k=3, the correlation between groups. The k represents the consensus matrices. (B) Consensus clustering CDF for k=2-10. (C) Relative change in area under CDF curve for k=2-10. (D) Differential DFS of patients with HCC in the three clusters. HCC, hepatocellular carcinoma; LIHC, Liver HCC; CDF, cumulative distribution function; DFS, disease-free survival.

significantly associated with OS. Increased expression levels of HNRNPA2B1, IGF2BP3, METTL3, WTAP, YTHDF1 and YTHDF2 indicated poorer OS and DFS rates in patients with HCC (Fig. S4). Applying the LASSO analysis, in which the selected m6A RNA methylation regulators were required to appear 900 times out of 1,000 repetitions, four m6A RNA methylation regulators, namely IGF2BP3, YTHDF1, YTHDF2 and METTL3, were selected. The distribution of the risk score was different among different clusters (Mann-Whitney U test; $\mathrm{P}=0.0013$; data not shown), and cluster 2, which was associated with a poorer DFS (Fig. 4D), had the highest risk score. To further investigate the prognostic role of the four-gene risk signature, patients with HCC were assigned into groups based on high- or low-risk scores using the cut-off value obtained using the survminer package, and it was observed that the high-risk group had a shorter DFS rate compared with the low-risk group (Fig. 5A). The AUC values of 1, 2 and 3 years were $0.783,0.720$ and 0.701 , respectively (Fig. 5C). The efficacy of the classifier was further evaluated using OS, and the results were similar to those for DFS (Fig. 5B). The AUC values of 1,2 and 3 years were $0.745,0.785$ and 0.794 , respectively (Fig. 5D). Similarly, patients with HCC in the meta-GEO and ICGC cohorts were divided into high- and low-risk groups, the high-risk group of the meta-GEO cohort exhibited worse OS and DFS rates compared with the low-risk group (Fig. S5A-D), and the high-risk group of the ICGC cohort (recurrence time was not collected) exhibited worse OS rates compared with the low-risk group (Fig. S5E and F).

m6A-based prognosis classifier and clinicopathological characteristics in patients with $H C C$. The heat map in Fig. 6A shows the expression levels of the four selected m6A RNA methylation regulators and the clinicopathological variables in the high- and low-risk groups. There was no 
A Prognostic value of m6A genes associated with DFS
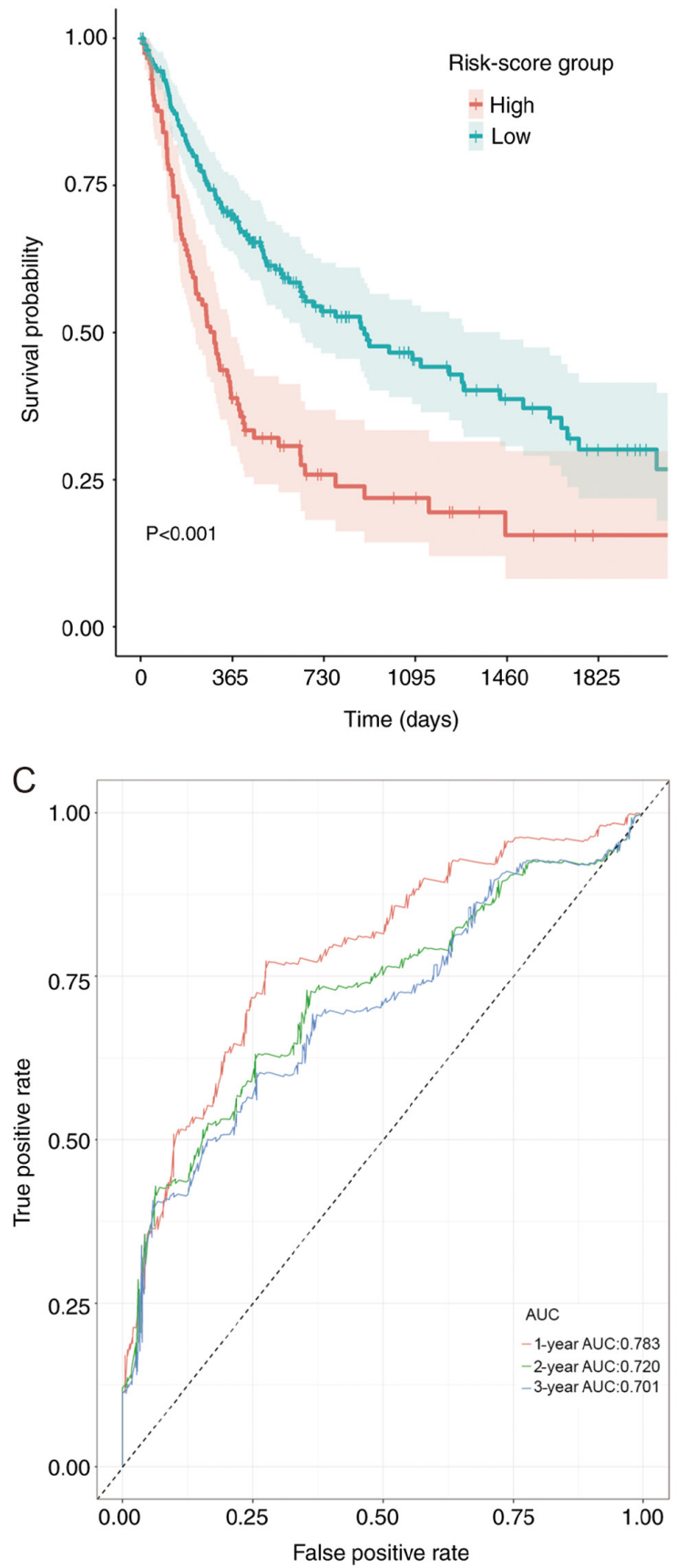

B Prognostic value of $\mathrm{m} 6 \mathrm{~A}$ genes associated with OS
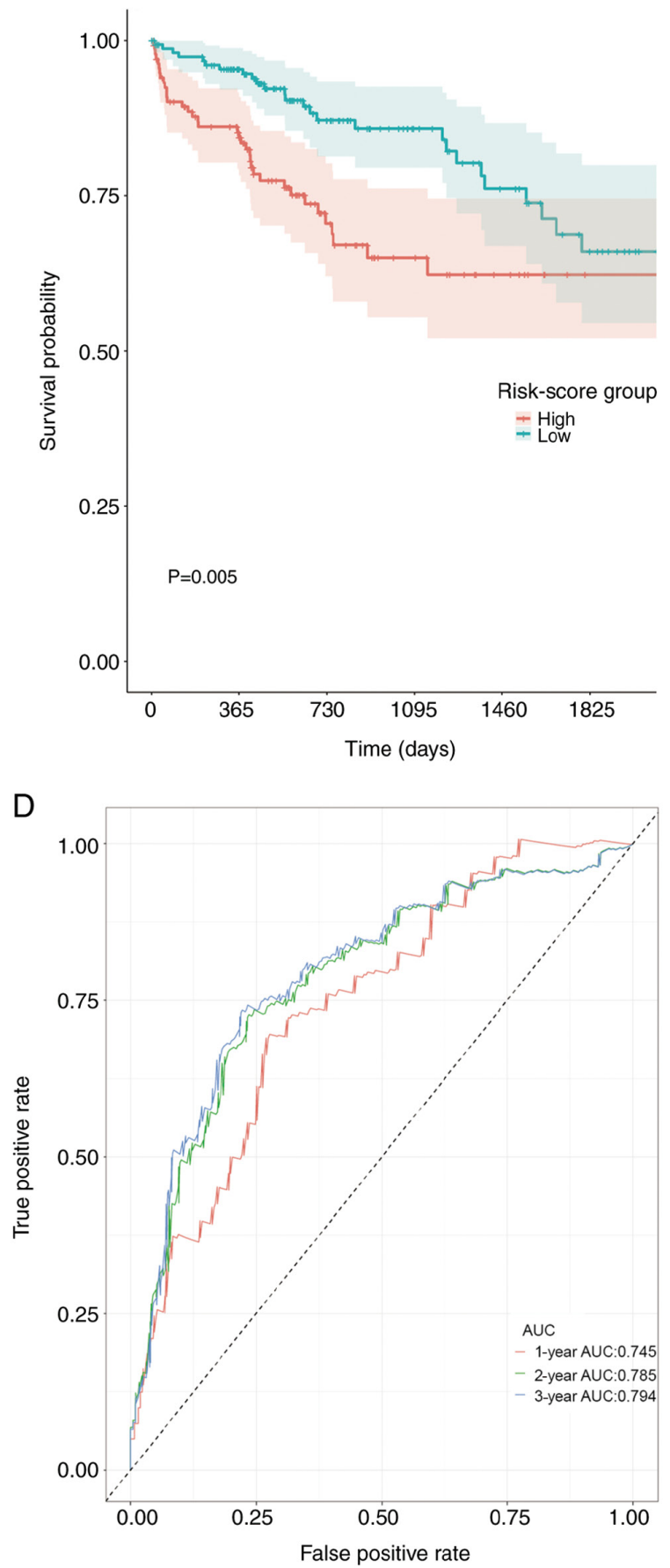

Figure 5. Kaplan-Meier curves for (A) DFS and (B) OS. Receiver operating characteristic curves for (C) DFS and (D) OS in high- and low-risk groups. DFS, disease-free survival; OS, overall survival; AUC, area under the curve; m6A, N6-methyladenosine.

marked difference between the two groups regarding sex and age. Similar to their association with prognosis, the low-risk group, with lower expression levels of the four genes, was significantly associated with lower $\mathrm{T}$ stage and pathological grade (Fig. 6B and C). Univariate and multivariate Cox regression analyses were performed to evaluate whether the prognostic signature-based risk score was an independent factor for prognosis. When the m6A signature was evaluated as a continuous variable with the Cox regression model, the univariate and multivariate analyses revealed that the stage and risk score were significantly associated with OS (Table I).
These results suggested that the risk signature may be a risk factor for HCC and may independently predict the prognosis of patients with HCC.

Differential biological signaling pathways, somatic mutation landscape and methylation-driven genes between the HCC risk score subtypes. To further identify the potential biological processes of the risk score subtypes, GSEA comparing the high- and low-risk groups was performed. The results revealed that the samples in the high-risk group were enriched in 'regulation of transcription' and 'immune system development', 

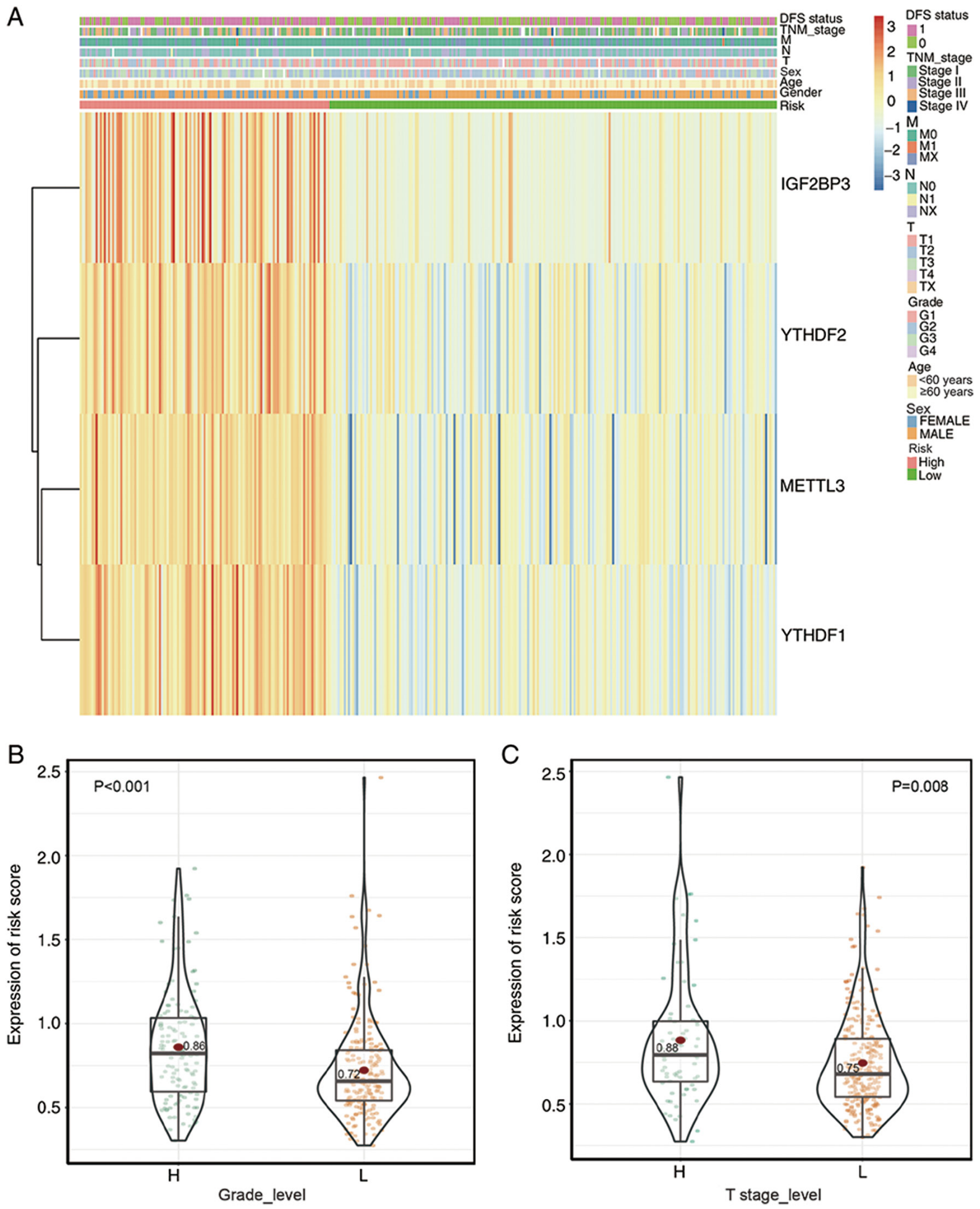

Figure 6. Characteristics of risk signature subtypes. (A) Heat map showing the clinical characteristics of the high- and low-risk groups. Patients were divided into high- and low-risk groups according to the optimal cut-off value of risk scores. (B and C) Significant differences were observed for (B) pathological grade and (C) T stage between the high- and low-risk groups. T stage, tumor stage; DFS, disease-free survival; TNM, Tumor-Node-Metastasis; H, high; L, low.

while the samples in the low-risk group were enriched in 'lipid biosynthetic process' (Fig. 7).

To identify the associations between the distributions of somatic alterations and the HCC risk score subtypes, 526 genes were identified with shared mutations between the high- and low-risk groups. Specifically, a missense mutation in TP53 and FLG was predominantly observed in the high-risk group, while the mutation frequency of ALB was higher in the low-risk group compared with in the high-risk group (Fig. 8).

Methylation-driven genes are genes with different degree of methylation and expression between different groups. After downloading and processing the methylation data, 569 methylation-driven genes associated with risk score 
Table I. Univariate and multivariate regression analysis of the association between the risk score and clinicopathological features with overall survival.

\begin{tabular}{|c|c|c|c|c|c|c|}
\hline \multirow[b]{2}{*}{ Factor } & \multicolumn{3}{|c|}{ Univariate analysis } & \multicolumn{3}{|c|}{ Multivariate analysis } \\
\hline & HR & P-value & $95 \% \mathrm{CI}$ & HR & P-value & $95 \% \mathrm{CI}$ \\
\hline Age ( $\geq 60$ vs. $<60$ years) & 1.11 & 0.492 & $0.83-1.48$ & 1.24 & 0.177 & $0.91-1.68$ \\
\hline Sex (male vs. female) & 0.91 & 0.531 & $0.67-1.23$ & 0.98 & 0.920 & $0.71-1.37$ \\
\hline $\begin{array}{l}\text { Pathological grade } \\
\text { (G3/G4 vs. G1/G2) }\end{array}$ & 1.10 & 0.543 & $0.81-1.48$ & 0.95 & 0.767 & $0.69-1.32$ \\
\hline Risk score (high vs. low) & 3.00 & $<0.001$ & $2.01-4.47$ & 2.51 & $<0.001$ & $1.59-3.97$ \\
\hline Tumor stage (III/IV vs. I/II) & 2.08 & $<0.001$ & $1.49-2.89$ & 1.79 & 0.001 & $1.26-2.54$ \\
\hline
\end{tabular}

HR, hazard ratio; TNM, Tumor-Node-Metastasis.

Table II. Methylation-driven genes associated with risk score subtypes of hepatocellular carcinoma.

A, Top 10 hypermethylated genes

\begin{tabular}{lc}
\hline Gene & $\log F C$ \\
\hline FMO3 & 0.558359143 \\
RBP5 & 0.550688961 \\
AKR7L & 0.546025322 \\
SLC2A2 & 0.527909372 \\
SLC27A2 & 0.451295029 \\
CFHR5 & 0.428486584 \\
CD14 & 0.416272718 \\
ACADL & 0.411132983 \\
SERPINC1 & 0.410346926 \\
APOC3 & 0.401360659 \\
\hline
\end{tabular}

B, Top 10 hypomethylated genes

\begin{tabular}{lc}
\hline Gene & $\log F C$ \\
\hline SP5 & -0.684538772 \\
BMP4 & -0.677013955 \\
FOXD3 & -0.641820658 \\
EVI2A & -0.600077451 \\
ZNF702P & -0.570013961 \\
FOXE1 & -0.560776731 \\
LRFN4 & -0.545665424 \\
AIM2 & -0.545136870 \\
LTC4S & -0.499335824 \\
TMEFF1 & -0.495389988 \\
\hline
\end{tabular}

FC, fold-change.

subtypes were screened via the MethylMix R package (Table II). Among these genes, 461 genes (81.02\%) were hypomethylated and the remaining 108 genes (18.98\%) were hypermethylated.

\section{Discussion}

The occurrence and development of HCC is a multi-step complex process involved with genetic or epigenetic factors $(31,32)$. Therefore, elucidating the underlying molecular events accounting for the tumorigenesis, diagnosis and precise individual therapy of HCC remain the greatest challenges. Previous studies have demonstrated that m6A affects the epigenetic regulation of RNA, including mRNA stability (33), alternative splicing (34) and microRNA biogenesis (35), which in turn regulate gene expression. The dysregulation of m6A genes affects the pathogenesis of a variety of human diseases, including obesity, neuronal disorders and immunological diseases, as well as promoting the initiation, expansion and progression of malignancies, including $\operatorname{HCC}(21,36,37)$. Previous studies (38-40) on mRNA m6A modification have associated the methylation levels of m6A with the intracellular writing and erasing genes, while the regulatory functions of methylation sites in biological processes is performed by protein molecules that read gene expression (41). Therefore, in tumors, both m6A-associated genes and protein expression levels may become potential diagnostic markers for tumor molecular diagnosis and potential targets for molecular targeted therapies.

The abnormal methylation of m6A mRNA has exhibited prognostic value in multiple types of tumor, such as cervical cancer (42), acute myeloid leukemia (43) and pancreatic cancer (44). Considering the importance of m6A modification in $\mathrm{HCC}$, it can be reasonably speculated that m6A-associated genes may have broad prospects in the prognostic evaluation of HCC, and that using a multigene signature generated using various algorithms may improve the prognostic prediction in patients with HCC compared with using a single molecule.

The risk model created in the present study consisted of four m6A-associated genes, and the risk score was an independent prognostic marker according to the multivariate analysis. In terms of validity and reliability, the four-gene signature performed well even in the external validation datasets. Furthermore, the high- and low-risk HCC groups presented with different significantly mutated genes, of which TP53 was markedly mutated in the high-risk HCC group compared with in the low-risk group. The association between the TP53 


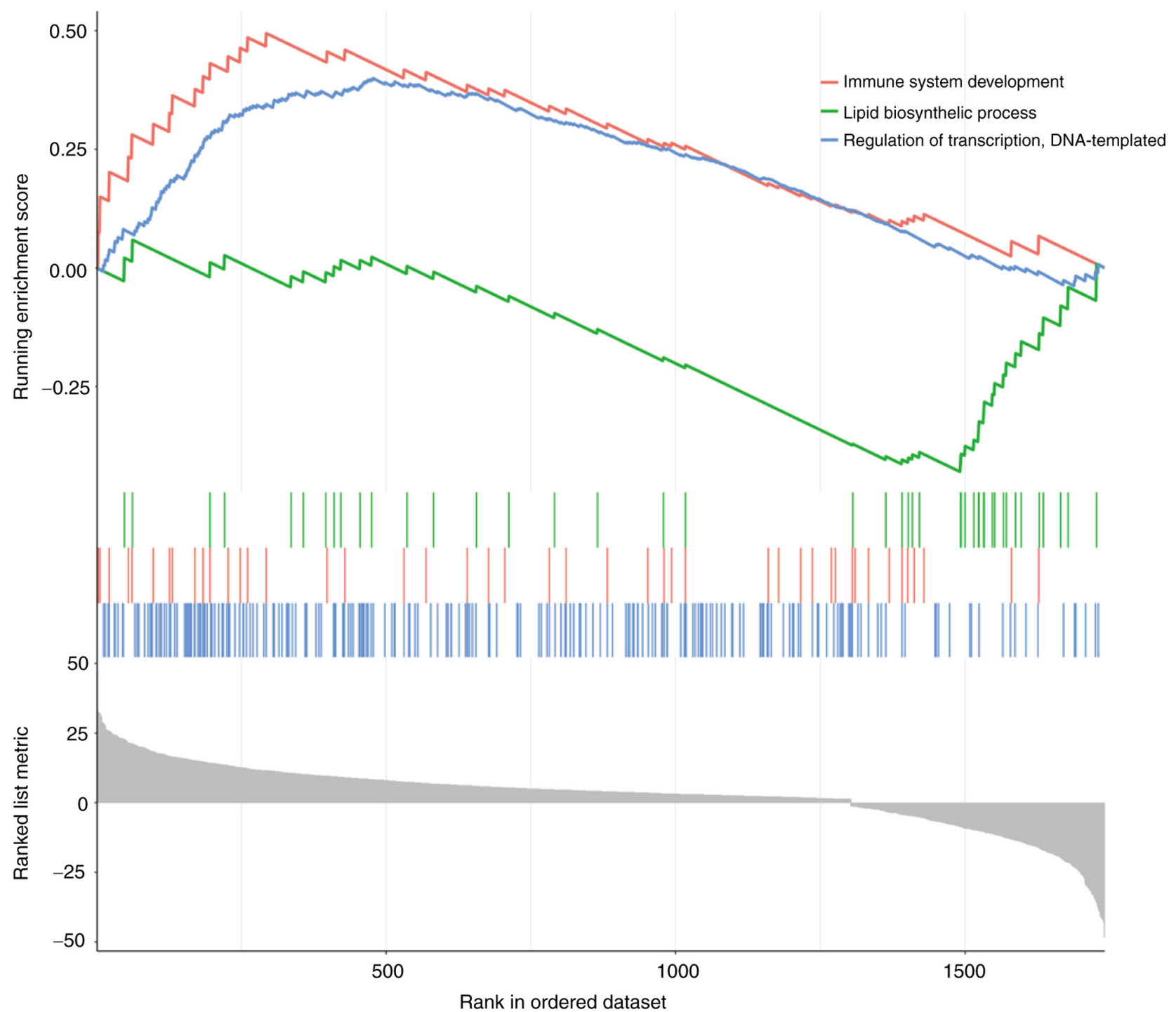

Figure 7. Differential signaling pathways between the hepatocellular carcinoma risk score subtypes. The gene set 'regulation of transcription' and 'immune system development' were enriched in the high-risk group, while 'lipid biosynthetic process' was enriched in the risk-low group.
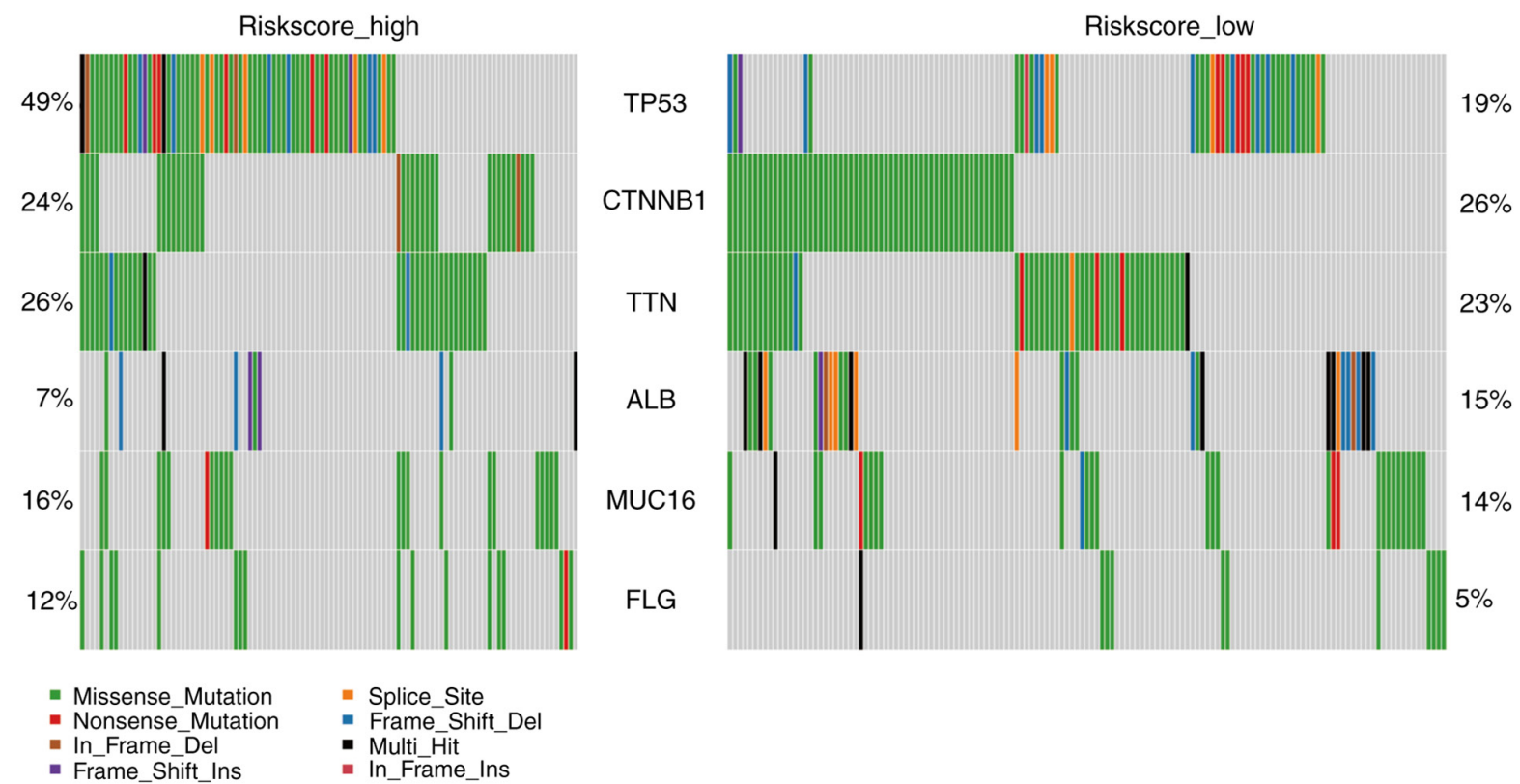

Figure 8. Genetic alteration between the risk score subtypes. Oncoprint shows variant mutated genes associated with risk score subtypes. 
signaling pathway and YTHDF2 expression has been previously reported (45), suggesting that the high-risk HCC group with increased TP53 mutations may be more likely to be involved in the activation of cancer signaling pathways. In addition, the identified METTL3/RDM1/TP53/ERK signaling pathway (46) may help to clarify the potential association of TP53 mutation and the risk signature in the present study. In the present study, integrative analysis by mRNA expression and promoter $\mathrm{CpG}$ islands methylation manifested a broad spectrum of gene silencing in the high-risk HCC group compared with that in the low-risk HCC group. These data may provide a new perspective to study the mechanism of m6A modulation.

Signaling pathways involved in the regulation of transcription, immune system development and lipid biosynthesis were differentially enriched in the phenotypes of the risk score in the present study. IGF2BPs (including IGF2BP1/2/3) promote the stability and storage of their target mRNAs (such as MYC) in an m6A-dependent manner under normal and stress conditions, and therefore affect gene expression output (47). Moreover, the $\mathrm{K}$ homology domains of IGF2BPs are required for their recognition of m6A and are critical for their oncogenic functions (48). YTHDF2 mainly regulates mRNA stability (49), and acts as a tumor-inhibiting factor in HCC $(50,51)$. YTHDF2 deficiency promotes HCC growth, vasculature remodeling and metastasis via a potential mechanism that involves the reprogramming of the epi-transcriptome under hypoxia $(50,52)$. Although the four genes in the current gene signature have not been reported to be associated with dyslipidemia, YTHDC2 may bind to the mRNA of lipogenic genes, including sterol regulatory element-binding protein 1c, fatty acid synthase, stearoyl-CoA desaturase 1 and acetyl-CoA carboxylase 1, to decrease their mRNA stability and inhibit gene expression (53). RNA m6A modification regulates anti-tumor immunity response via YTHDF1, which regulates tumorigenicity and cancer stem cell-like activity in HCC (48). Transcripts encoding lysosomal proteases are marked by $\mathrm{m} 6 \mathrm{~A}$ and recognized by YTHDF1; binding of YTHDF1 to these transcripts increases the translation of lysosomal cathepsins in dendritic cells, and inhibition of cathepsins markedly enhances cross-presentation of wild-type dendritic cells (54). Furthermore, the therapeutic efficacy of programmed death-ligand 1 checkpoint blockade is enhanced in Ythdf $1^{-/-}$mice, implicating YTHDF1 as a potential therapeutic target in anticancer immunotherapy (54).

The present study presents some limitations. Firstly, although several independent external validations were performed in the present study, it was difficult to consider all variations among patients from different geographical regions, since tissues and data were retrospectively collected from publicly available databases. In addition, other key clinical pathological features, such as surgical procedures, the number of lymph nodes and $\alpha$-fetoprotein levels, were not included. Secondly, the mechanism and association between the risk score subtypes and single nucleotide polymorphisms and DNA methylation require further study. Thirdly, IGF2BP3, YTHDF2, METTL3 and YTHDF1 were indicated to be associated with HCC. However, the mechanism underlying the higher prognostic efficiency of the combination of these molecules, as determined from three independent cohorts, remains unclear. Finally, the expression levels and prognostic role of these four genes require further validation by well-designed, prospective, multicenter studies.
Overall, the present study comprehensively analyzed the associations between the mRNA expression levels of m6A regulators with the initiation, development and prognosis of HCC. Notably, a robust four-gene prognostic signature that was significantly associated with the OS of patients with HCC was constructed and validated in independent HCC cohorts, suggesting that the present prognostic signature may act as a promising biomarker for predicting the prognosis of patients with HCC. Additionally, it may serve as a prognostic classifier for clinical decision-making for the accurate prognosis prediction, treatment and follow-up scheduling.

\section{Acknowledgements}

Not applicable.

\section{Funding}

The present study was supported by the National Natural Science Foundation of China (grant nos. 81301301 and 81601579), the Chen Xiao-ping Foundation for the Development of Science and Technology of Hubei Province (grantno.CXPJJH11800001-2018203) and the Natural Science Foundation of Hubei province (grant no. 2018CFB553).

\section{Availability of data and materials}

The datasets generated and/or analyzed during the current study are available in the National Cancer Institute Genomic Data Commons (https://gdc.cancer.gov/), Gene Expression Omnibus (https://www.ncbi.nlm.nih.gov/geo/), International Cancer Genome Consortium (https://icgc.org/) and Human Protein Atlas databases (http://www.proteinatlas.org/).

\section{Authors' contributions}

QJ and ZG designed the study. NH and CZ performed data acquisition and collected the literature. PZ and QR performed data analysis and interpretation, and drafted the manuscript. QJ critically revised the manuscript for important intellectual content. All authors read and approved the final version of the manuscript.

\section{Ethics approval and consent to participate}

Not applicable.

\section{Patient consent for publication}

Not applicable.

\section{Competing interests}

The authors declare that they have no competing interests.

\section{References}

1. Siegel RL, Miller KD and Jemal A: Cancer statistics, 2016. CA Cancer J Clin 66: 7-30, 2016.

2. Thorgeirsson SS and Grisham JW: Molecular pathogenesis of human hepatocellular carcinoma. Nat Genet 31:339-346, 2002. 
3. Jung HI, Jeong D, Ji S, Ahn TS, Bae SH, Chin S, Chung JC, Kim HC, Lee MS and Baek MJ: Overexpression of PD-L1 and PD-L2 is associated with poor prognosis in patients with hepatocellular carcinoma. Cancer Res Treat 49: 246-254, 2017.

4. Gish RG, Porta C, Lazar L, Ruff P, Feld R, Croitoru A, Feun L, Jeziorski K, Leighton J, Gallo J and Kennealey GT: Phase III randomized controlled trial comparing the survival of patients with unresectable hepatocellular carcinoma treated with nolatrexed or doxorubicin. J Clin Oncol 25: 3069-3075, 2007.

5. Llovet JM, Brú C and Bruix J: Prognosis of hepatocellular carcinoma: The BCLC staging classification. Seminars Liver Disease 19: 329-338, 1999.

6. Yoo JJ, Chung GE, Lee JH, Nam JY, Chang Y, Lee JM, Lee DH, Kim HY, Cho EJ, Yu SJ, et al: Sub-classification of advanced-stage hepatocellular carcinoma: A cohort study including 612 patients treated with sorafenib. Cancer Res Treat 50: 366-373, 2018.

7. Yoon KJ, Ringeling FR, Vissers C, Jacob F, Pokrass M, Jimenez-Cyrus D, Su Y, Kim NS, Zhu Y, Zheng L, et al: Temporal control of mammalian cortical neurogenesis by $\mathrm{m}(6) \mathrm{A}$ methylation. Cell 171: 877-889.e17, 2017.

8. Schibler U, Kelley DE and Perry RP: Comparison of methylated sequences in messenger RNA and heterogeneous nuclear RNA from mouse L cells. J Mol Biol 115: 695-714, 1977.

9. Wei CM and Moss B: Nucleotide sequences at the N6-methyladenosine sites of HeLa cell messenger ribonucleic acid. Biochemistry 16: 1672-1676, 1977.

10. Desrosiers R, Friderici K and Rottman F: Identification of methylated nucleosides in messenger RNA from Novikoff hepatoma cells. Proc Natl Acad Sci USA 71: 3971-3975, 1974.

11. Wei CM, Gershowitz A and Moss B: Methylated nucleotides block 5 ' terminus of HeLa cell messenger RNA. Cell 4: 379-386, 1975.

12. Zhao BS, Roundtree IA and He C: Post-transcriptional gene regulation by mRNA modifications. Nat Rev Mol Cell Biol 18 : 31-42, 2017.

13. Meyer KD, Saletore Y, Zumbo P, Elemento O, Mason CE and Jaffrey SR: Comprehensive analysis of mRNA methylation reveals enrichment in 3' UTRs and near stop codons. Cell 149: $1635-1646,2012$

14. Helm M and Motorin Y: Detecting RNA modifications in the epitranscriptome: Predict and validate. Nat Rev Genet 18 275-291, 2017.

15. Pinello N, Sun S and Wong JJ: Aberrant expression of enzymes regulating $\mathrm{m} 6 \mathrm{~A}$ mRNA methylation: Implication in cancer. Cancer Biol Med 15: 323-334, 2018.

16. Batista PJ: The RNA modification N(6)-methyladenosine and its implications in human disease. Genomics Proteomics Bioinformatics 15: 154-163, 2017.

17. Zhou S, Bai ZL, Xia D, Zhao ZJ, Zhao R, Wang YY and Zhe H: FTO regulates the chemo-radiotherapy resistance of cervical squamous cell carcinoma (CSCC) by targeting $\beta$-catenin through mRNA demethylation. Mol Carcinog 57: 590-597, 2018

18. Liu ZX, Li LM, Sun HL and Liu SM: Link between m6A modification and cancers. Front Bioeng Biotechnol 6: 89, 2018.

19. Cai X, Wang X, Cao C, Gao Y, Zhang S, Yang Z, Liu Y, Zhang X, Zhang W and Ye L: HBXIP-elevated methyltransferase METTL3 promotes the progression of breast cancer via inhibiting tumor suppressor let-7g. Cancer Lett 415: 11-19, 2018.

20. Taketo K, Konno M, Asai A, Koseki J, Toratani M, Satoh T, Doki Y, Mori M, Ishii H and Ogawa K: The epitranscriptome m6A writer METTL 3 promotes chemo-and radioresistance in pancreatic cancer cells. Int J Oncol 52: 621-629, 2018.

21. Cheng X, Li M, Rao X, Zhang W, Li X, Wang L and Huang G: KIAA1429 regulates the migration and invasion of hepatocellular carcinoma by altering m6A modification of ID2 mRNA Onco Targets Ther 12: 3421-3428, 2019.

22. Mayakonda A, Lin DC, Assenov Y, Plass C and Koeffler HP Maftools: Efficient and comprehensive analysis of somatic variants in cancer. Genome Res 28: 1747-1756, 2018

23. Gevaert O: MethylMix: An R package for identifying DNA methylation-driven genes. Bioinformatics 31: 1839-1841, 2015.

24. Roessler S, Jia HL, Budhu A, Forgues M, Ye QH, Lee JS, Thorgeirsson SS, Sun Z, Tang ZY, Qin LX and Wang XW: A unique metastasis gene signature enables prediction of tumor relapse in early-stage hepatocellular carcinoma patients. Cancer Res 70: 10202-10212, 2010

25. Grinchuk OV, Yenamandra SP, Iyer R, Singh M, Lee HK, Lim KH, Chow PK and Kuznetsov VA: Tumor-adjacent tissue co-expression profile analysis reveals pro-oncogenic ribosomal gene signature for prognosis of resectable hepatocellular carcinoma. Mol Oncol 12: 89-113, 2018.
26. Wilkerson D M, Hayes and Neil D: ConsensusClusterPlus: a class discovery tool with confidence assessments and item tracking. Bioinformatics, 26, 1572-1573, 2010.

27. Lacny S, Wilson T, Clement F, Roberts DJ, Faris PD, Ghali WA and Marshall DA: Kaplan-Meier survival analysis overestimates the risk of revision arthroplasty: A meta-analysis. Clin Orthop Relat Res 473: 3431-3442, 2015.

28. Tibshirani R: The lasso method for variable selection in the Cox model. Stat Med 16: 385-395, 1997.

29. American Joint Committee On Cancer: AJCC 7th edition Cancer Staging Manual, 2009. https://cancerstaging.org/references-tools/ deskreferences/Documents/AJCC\%207th\%20Ed\%20Cancer\%20 Staging\%20Manual.pdf. Accessed December 3, 2019.

30. Monti S, Tamayo P, Mesirov J and Golub T: Consensus clustering: A resampling-based method for class discovery and visualization of gene expression microarray data. Machine Learn 52: 91-118, 2003.

31. Sun LY, Li XY and Sun ZW: Progress of epigenetics and its therapeutic application in hepatocellular carcinoma. Yi Chuan 37: 517-527, 2015 (In Chinese)

32. Feo F, Frau M, Tomasi ML, Brozzetti S and Pascale RM: Genetic and epigenetic control of molecular alterations in hepatocellular carcinoma. Exp Biol Med (Maywood) 234: 726-736, 2009.

33. Geula S, Moshitch-Moshkovitz S, Dominissini D, Mansour AA, Kol N, Salmon-Divon M, Hershkovitz V, Peer E, Mor N, Manor YS, et al: m6A mRNA methylation facilitates resolution of naïve pluripotency toward differentiation. Science 347 1002-1006, 2015

34. Lence T, Akhtar J, Bayer M, Schmid K, Spindler L, Ho CH, Kreim N, Andrade-Navarro MA, Poeck B, Helm M and Roignant JY: $\mathrm{m}^{6} \mathrm{~A}$ modulates neuronal functions and sex determination in Drosophila. Nature 540: 242-247, 2016.

35. Alarcón CR, Lee H, Goodarzi H, Halberg N and Tavazoie SF: N 6-methyladenosine marks primary microRNAs for processing. Nature 519: 482-485, 2015.

36. He L, Li H, Wu A, Peng Y, Shu G and Yin G: Functions of N6-methyladenosine and its role in cancer. Mol Cancer 18: 176, 2019.

37. Chen Y, Peng C, Chen J, Chen D, Yang B, He B, Hu W, Zhang Y, Liu H, Dai L, et al: WTAP facilitates progression of hepatocellular carcinoma via m6A-HuR-dependent epigenetic silencing of ETS1. Mol Cancer 18: 127, 2019.

38. Meyer KD and Jaffrey SR: The dynamic epitranscriptome: N6-methyladenosine and gene expression control. Nat Rev Mol Cell Biol 15: 313-326, 2014.

39. Shi H, Wei J and He C: Where, when, and how: Context-dependent functions of RNA methylation writers, readers, and erasers. Mol Cell 74: 640-650, 2019.

40. Zaccara S, Ries RJ and Jaffrey SR: Reading, writing and erasing mRNA methylation. Nat Rev Mol Cell Biol 20: 608-624, 2019.

41. Yang Y, Hsu PJ, Chen YS and Yang YG: Dynamic transcriptomic $\mathrm{m}^{6} \mathrm{~A}$ decoration: Writers, erasers, readers and functions in RNA metabolism. Cell Res 28: 616-624, 2018

42. Wang X, Li Z, Kong B, Song C, Cong J, Hou J and Wang S: Reduced $\mathrm{m}^{6} \mathrm{~A}$ mRNA methylation is correlated with the progression of human cervical cancer. Oncotarget 8: 98918-98930, 2017.

43. Kwok CT, Marshall AD, Rasko JE and Wong JJ: Genetic alterations of $\mathrm{m}(6)$ A regulators predict poorer survival in acute myeloid leukemia. J Hematol Oncol 10: 39, 2017.

44. Cho SH, Ha M, Cho YH, Ryu JH, Yang K, Lee KH, Han ME, Oh SO and Kim YH: ALKBH5 gene is a novel biomarker that predicts the prognosis of pancreatic cancer: A retrospective multicohort study. Ann Hepatobiliary Pancreat Surg 22: 305-309, 2018.

45. Li Y, Xiao J, Bai J, Tian Y, Qu Y, Chen X, Wang Q, Li X, Zhang Y and $\mathrm{Xu} \mathrm{J}$ : Molecular characterization and clinical relevance of $\mathrm{m}^{6} \mathrm{~A}$ regulators across 33 cancer types. Mol Cancer 18: 137, 2019.

46. Chen SL, Liu LL, Wang CH, Lu SX, Yang X, He YF, Zhang CZ and Yun JP: Loss of RDM1 enhances hepatocellular carcinoma progression via p53 and Ras/Raf/ERK pathways. Mol Oncol 14: 373-386, 2020

47. Huang H, Weng H, Sun W, Qin X, Shi H, Wu H, Zhao BS, Mesquita A, Liu C, Yuan CL, et al: Recognition of RNA $\mathrm{N}(6)$-methyladenosine by IGF2BP proteins enhances mRNA stability and translation. Nat Cell Biol 20: 285-295, 2018.

48. Zhao X, Chen Y, Mao Q, Jiang X, Jiang W, Chen J, Xu W, Zhong L and Sun X: Overexpression of YTHDF1 is associated with poor prognosis in patients with hepatocellular carcinoma. Cancer Biomark 21: 859-868, 2018.

49. Wang $\mathrm{X}, \mathrm{Lu} \mathrm{Z}$, Gomez A, Hon GC, Yue Y, Han D, Fu Y, Parisien M, Dai Q, Jia G, et al: N6-methyladenosine-dependent regulation of messenger RNA stability. Nature 505: 117-120, 2014. 
50. Hou J, Zhang H, Liu J, Zhao Z, Wang J, Lu Z, Hu B, Zhou J, Zhao Z, Feng M, et al: YTHDF2 reduction fuels inflammation and vascular abnormalization in hepatocellular carcinoma. Mol Cancer 18: 163, 2019.

51. Zhong L, Liao D, Zhang M, Zeng C, Li X, Zhang R, Ma H and Kang T: YTHDF2 suppresses cell proliferation and growth via destabilizing the EGFR mRNA in hepatocellular carcinoma. Cancer Lett 442: 252-261, 2019.

52. Chen M, Wei L, Law CT, Tsang FH, Shen J, Cheng CL, Tsang LH, Ho DW, Chiu DK, Lee JM, et al: RNA N6-methyladenosine methyltransferase-like 3 promotes liver cancer progression through YTHDF2-dependent posttranscriptional silencing of SOCS2. Hepatology 67: 2254-2270, 2018.
53. Zhou B, Liu C, Xu L, Yuan Y, Zhao J, Zhao W, Chen Y, Qiu J, Meng M, Zheng Y, et al: $\mathrm{N}^{6}$-methyladenosine reader protein Ythdc2 suppresses liver steatosis via regulation of mRNA stability of lipogenic genes. Hepatology: Mar 9, 2020 (Epub ahead of print). doi: 10.1002/hep.31220.

54. Han D, Liu J, Chen C, Dong L, Liu Y, Chang R, Huang X, Liu Y, Wang J, Dougherty U, et al: Anti-tumour immunity controlled through mRNA m(6)A methylation and YTHDF1 in dendritic cells. Nature 566: 270-274, 2019.

This work is licensed under a Creative Commons Attribution-NonCommercial-NoDerivatives 4.0 International (CC BY-NC-ND 4.0) License. 